\title{
A RARE CASE OF SYSTEMIC SCLEROSIS FOLLOWING THYMECTOMY IN A PATIENT WITH MYASTHENIA GRAVIS
}

Marcel Mathias Villaça ${ }^{1, \star}$, Júlia Boechat Farani ${ }^{1}$, Erika Biegelmeyer ${ }^{1}$, Evelise Mileski do Amaral Berlet ${ }^{1}$, Marília Voges de Souza ${ }^{1}$, Diego Ustárroz Cantali ${ }^{1}$

1.Pontifícia Universidade Católica do Rio Grande do Sul, Porto Alegre (RS), Brazil.

*Corresponding author:marcel_mv@hotmail.com.br

\section{BACKGROUND}

Thymectomy is a well-known treatment for myasthenia gravis (MG). Literature is sparse on systemic sclerosis (SS) in overlap with MG, but cases of post-thymectomy autoimmune diseases have been reported.

\section{CASE REPORT}

We report the case of a 67-year-old white female patient, with a previous diagnosis of MG since June 2020, presenting with progressive, asymmetric fluctuating weakness. On electromyography, there was postsynaptic dysfunction of the neuromuscular junction plus axonal, chronic and asymmetric sensorimotor polyneuropathy. Chest computed tomography revealed an anterior mediastinal mass compatible with thymoma. Anticholinesterase therapy was initiated with pyridostigmine and thymectomy was performed 4 months later. Pathology was compatible with thymoma with a mixed medullary (70\%) and cortical pattern (B1 20\% and B2 10\%). Patient failed extubation at immediate postoperatory period due to ventilatory fatigue, suggesting disease activity. Intravenous immune globulin at a dose of $2 \mathrm{mg} / \mathrm{kg}$ was administered for 2 days, and she was started on prednisone up to $60 \mathrm{mg} /$ day and azathioprine up to $150 \mathrm{mg} /$ day, with a good initial response. However, 3 months after thymectomy, she presented with myalgias, chronic inflammatory diarrhea, peripheral edema and arciform erythematous lesions in lower limbs. There was also rapidly progressive facial and chest telangiectasias, puffy fingers, Raynaud's phenomenon, labial thinning and dysphagia. She was admitted for investigation and laboratories revealed positive antinuclear antibodies with a title of 1:160 and a centromeric pattern, which was confirmed by positive anticentromere antibodies of 1:640. There was also a positive serology for hepatitis B virus (HBV) with high HBV DNA levels of 21,100,000 UI/mL $(7.32 \mathrm{log})$ by polymerase chain reaction. Esophageal transit scintigraphy showed significant retention in lower esophagus, suggesting abnormal esophageal motility. She was then diagnosed with SS in overlap with MG. Unfortunately, she developed an acute pulmonary embolism and a major fatal bleeding due to anticoagulation.

\section{CONCLUSION}

This is an intriguing case of SS in overlap with MG. Thymectomy may have contributed to the onset of SS, since the thymus plays an essential role in immune regulation. Genetic predisposition and a viral trigger might also have been implicated in co-occurrence of the diseases.

\section{KEYWORDS}

Systemic sclerosis, Myasthenia gravis, Thymectomy. 